\title{
WSGP 8
}

\section{A. K. Kwaśniewski}

On peculiarity of the $p=2$ case among the $p$-state Ising-like models.

In: Jarolím Bureš and Vladimír Souček (eds.): Proceedings of the Winter School "Geometry and Physics". Circolo Matematico di Palermo, Palermo, 1989. Rendiconti del Circolo Matematico di Palermo, Serie II, Supplemento No. 21. pp. [259]--264.

Persistent URL: http://dml.cz/dmlcz/701445

\section{Terms of use:}

(C) Circolo Matematico di Palermo, 1989

Institute of Mathematics of the Academy of Sciences of the Czech Republic provides access to digitized documents strictly for personal use. Each copy of any part of this document must contain these Terms of use.

This paper has been digitized, optimized for electronic delivery and stamped
with digital signature within the project $D M L-C Z$ : The Czech Digital Mathematics
Library http://project.dml.cz


ON PECULIARI'TY OF THE P=2 CASE AMONG THE P-STATE ISING-LIKE MODELS*

\section{A.K. Kwaśniewski}

Abstract

New inaications of the $\mathrm{p}=2$ case peculiarity among p-state lattice models are found while studing a relation between Rademacher and hyperbolic functions.

I. Introduction.

Consider the well known formula (Vieta)

$$
\frac{\sin x}{x}=\prod_{k=1}^{\infty} \cos \frac{x}{2^{k}} \quad 0 \neq x \in \mathbb{R}
$$

In [1] Mark Kac has shown how a specifically wise derivation of (V) reveals the stochastic properties of numbers via their dyadic representation.

At the same time - such representations are 1:1 related to properties of walsh functions which form a base of a specific example of maximally graded algebra [2] where

$$
\Gamma \equiv z_{2} \oplus \ldots \oplus z_{2} \oplus \ldots \equiv z\left(2^{\infty}\right)
$$

Algebras of the kind and generalized Walsh functions are already known to be of big importance for the theory of lattice spin systems with the grading group being in general that of $\mathrm{z}\left(\mathrm{p}^{\infty}\right)$;

$$
z\left(p^{\infty}\right)=z_{p} \oplus \cdots \oplus z_{p} \oplus \cdots
$$

as described in $[2,3]$.

*) Work performed under the exchange agreement between University of Rome and University of Wroclaw. 
The case $\mathrm{p}=2$ corresponds in the context to Ising model which is a very peculiar one (simply - it is solved!) among all potts models with $\mathrm{Z}\left(\mathrm{p}^{\infty}\right)$ grading group ( $\mathrm{p} \equiv$ prime number; once for all, in this note).

Question

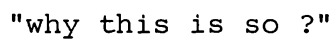

we shall try to trace further reasons for that apart from the ones discussed already in $[2,3,4]$

Procedure

For that to do, we shall try to generalize some of the very first observations of M. Kac in [1] and see why this or that is not possible.

Remark

We shall work rather with hypergeometric functions (generalized cosh functions $[2,4])$ than with Mikusinski's generalization of sin and cos trygonometry from $z_{2}$ to $z_{p}$ case, because partition functions of Potts models are polynomials in generalized cosh functions.

Result

The first results, however partial, serve as additional (to those found in $[2,4]$ ) indications "why this is so".

II. Rademacher and hyperbolic functions.

After recalling some prerequisities concerning real Rademacher step functions - a relation between them and hyperbolic function $f_{0}$ is found.

Def.

$$
\begin{array}{ll}
r_{0}, & r_{0}(x)=\left\{\begin{array}{cc}
1 & 0<x<\frac{1}{2} \\
-1 & \frac{1}{2}<x<1
\end{array} \quad ; r_{0}(x+1)=r_{0}(x) ;\right. \\
r_{n} ; & r_{n}(x)=r_{0}\left(2^{n} x\right) \quad
\end{array}
$$

Name

$$
\left\{r_{n}\right\}_{0}^{\infty} \text { - the set of Rademacher functions. }
$$

Observation 1.

$$
\left\{r_{n}\right\}_{0}^{\infty} \text { - the set of orthonormal functions. }
$$

Now let $\mathrm{x}_{1}:[0,1] \rightarrow \mathrm{z}_{2}$; 


$$
x \rightarrow x_{i}(x) ; \quad x=\sum_{i=1}^{\infty} \frac{x_{i}(x)}{2^{i}}
$$

where, in the case of two dyadic representation of $x$, allways the finite one is chosen.

Then one has an

Observation 2 .

$$
[0,1] \ni x ; \quad r_{n}(x) \equiv 1-2 x_{n}(x) \equiv(-1)^{x_{n}} \text {. }
$$

We now extend the definition of Rademacher functions from the case $\mathrm{p}=2$ to arbitrary prime $\mathrm{p}$.

Def.

Def.

$$
\begin{aligned}
& r_{k}(x)=\omega_{k}^{x_{k}} \quad ; \quad \text { where } \\
& x=\sum_{k=1}^{\infty} \frac{x_{k}}{p^{k}} \quad ; \quad x_{k} \in z_{p} \text { and } \omega=\exp \left\{\frac{i 2 \pi}{p}\right\} \text {. }
\end{aligned}
$$

Name

$\left\{r_{k}\right\}_{1}^{\infty}-p$-Rademacher functions.

\section{Observation}

These functions form a set of orthonormal function.

Observation 3 .

$$
\begin{array}{r}
{[0,1] \exists x \quad r_{n}(x)=\sum_{i \in Z_{p}} \omega^{i}(p-1)\left(x_{n}-1\right) \cdots \ldots\left(x_{n}-i+1\right)} \\
\cdot\left(x_{n}-i-1\right) \cdots \cdots\left(x_{n}-p+1\right)
\end{array}
$$

where $z_{p}$ is considered now to be the field.

$\underline{\text { Proof }}$

It is enough to take into account:

a) Wilson's theorem $\Leftrightarrow(\mathrm{p}-1) !=\mathrm{p}-1$ (modp) iff $\mathrm{p}$-prime.

b) $(-1) \cdot(-2) \cdot \ldots \cdot(-p+1)=(p-1) !(\operatorname{modp})$.

Observation 4 .

2)

$r_{k}$ is a linear function of $x_{k}$ iff $p=2$ (see Observation

Conseguence 1.

For $\mathrm{p}=2$ one has

$$
\lim _{n \rightarrow \infty} \int_{0}^{1} \exp \left(i x \sum_{k=1}^{n} \frac{r_{k}(t)}{2^{k}}\right)=\frac{\sin x}{x}
$$


what leads to $(V)$, as at the same time

$$
\lim _{n \rightarrow \infty} \int_{0}^{1} \exp \left(\operatorname{ix} \sum_{k=1}^{n} \frac{r_{k}(t)}{2^{k}}\right)=\prod_{k=1}^{\infty} \cos \frac{x}{2^{k}}
$$

\section{Proof}

$$
\text { (see [1]). }
$$

Comment:

The second formula of Consequence 1 . exhibits the relation between 2-Rademacher functions and cos function.

This relation can be generalized to arbitrary p-prime.

Def.

$$
f_{i}(x)=\frac{1}{p} \sum_{k=0}^{p-1} \omega^{-k i} \exp \left\{\omega^{k} x\right\} \quad ; \quad x \in \mathbb{R}, \quad i \in z_{p}
$$

Name

$$
\begin{gathered}
\left\{f_{i}\right\}_{i \in Z_{p}} \Leftrightarrow \text { the set of hyperbolic functions (generalized cosh } \\
\text { functions) }
\end{gathered}
$$

Generalization 1. (first step)

$$
\int_{0}^{1} \exp \left\{\sum_{k=1}^{n} c_{k} r_{k}(t)\right\} d t=\prod_{k=1}^{n} f_{o}\left(c_{k}\right) ; \quad c_{k} \in \mathbb{R} \text {. }
$$

\section{Proof}

$$
\begin{aligned}
\int_{0}^{1} \exp \left\{\sum_{k=1}^{n} c_{k} r_{k}(t)\right\} d t & =\frac{1}{p^{n}} \sum_{\{1(k)\}} \exp \left\{\omega^{1(k)} c_{k}\right\}= \\
& =\prod_{k=1}^{n} \frac{1}{p} \sum_{1=1}^{p-1} \exp \left\{\omega^{1} c_{k}\right\} ;
\end{aligned}
$$

where

$$
\{l(k)\}_{1}^{n} \in z_{p} \oplus \ldots \oplus z_{p} \quad(n-\text { summands })
$$

Generalization 2. (second step)

Let us first define:

Def

$$
w["]:[0,1] \rightarrow[0,1]
$$




$$
\begin{aligned}
& {[0,1] \ni x \rightarrow w[" x "] \equiv \sum_{n=1}^{\infty} \frac{w\left[x_{n}\right]}{p^{n}} \text { where }} \\
& x=\sum_{n=1}^{\infty} \frac{x_{n}}{p^{n}} \text { and } w: z_{p} \rightarrow z_{p}
\end{aligned}
$$

Name

$$
\mathrm{w}[" \mathrm{n}=\mathrm{p} \text {-adic function on }[0,1] \text {. }
$$

Def

$$
\begin{aligned}
& \mathrm{w}_{\mathrm{k}}: \mathrm{z}_{\mathrm{p}} \rightarrow \mathrm{z}_{\mathrm{p}} ; \\
& \mathrm{z}_{\mathrm{p}} \ni \alpha \rightarrow \mathrm{w}_{\mathrm{k}}(\alpha) \equiv(\mathrm{p}-1) \cdot \alpha \cdot(\alpha-1) \cdots(\stackrel{\mathrm{v}}{\mathrm{v}}) \ldots(\alpha-\mathrm{k}) \cdot \ldots(\alpha-\mathrm{p}+1) ;
\end{aligned}
$$

where " $v$ " sign over factor $(\alpha-k)$ means: "the factor $(\alpha-k)$ is absent". Observation 5 .

$$
\sum_{n=1}^{\infty} \frac{r_{n}(x)}{p^{n}} \equiv \sum_{k=0}^{p-1} \omega^{k} w_{k}[" x "]
$$

Observation 6.

$$
\forall k \in \mathrm{z}_{\mathrm{p}} \quad \mathrm{w}_{\mathrm{k}}[" \mathrm{x} "] \equiv \mathrm{w}_{\mathrm{k}}[\mathrm{x}] \quad \text { iff. } \mathrm{p}=2 \text {. }
$$

Comment:

$$
\text { Observation } 6 \text { Observation } 4 \text {. }
$$

\section{Generalization}

$$
\int_{0}^{1} \exp \left\{x \sum_{k=0}^{p-1} w_{k}[" t "]\right\} d t=\prod_{s=1}^{\infty} f_{\circ}\left(\frac{x}{p}\right)
$$

III. Towards generalization of Vieta's formula.

In II we have produced, in a sense a "half of such a formula" i.e. we have generalized only the second formula from consequence 1. As linearity of $r_{n}$ in $x_{n}$ argument is crucial to have the left-hand side of (V) we feel hopeless in the case of $p>2$. However, in this respect we may proceed in another way; trying to generalize the formula (1.1) in [1] i.e. 


$$
\sin x=2^{n} \sin \frac{x}{2^{n}} \prod_{k=1}^{n} \cos \frac{x}{2^{k}}
$$

We do not feel it to be an easy thing for us, hence we take now $p=3$. For that case one has:

Lemma

$$
3 f_{\circ}(x) f_{1}(x) f_{2}(x)=\frac{1}{3}\left[f_{\circ}(3 x)-1\right]
$$

with $x \in A$ where $A$ is any associative algebra with unity; dimA $<\infty$.

The proof consists of just straightforward - though slightly tricky

- calculation.

In order to follow the scheme leading to $(S)$ in ordinary case; one should now know how to express also $f_{1}(3 x)$ and $f_{2}(3 x)$ in terms of $f_{0}(x), f_{1}(x)$ and $f_{2}(x)$.

Once this is known the (V) formula for the case of $p=3$ is at hand. Up to now however this is not known to us.

\section{REFERENCES}

[1] KAC M. "Statistical Independence in Probability Analysis and Number I'heory", 'The Carus Mathematical Monographs, The Mathematical Associacion of America, 1959

[2] KWÁ́SIEWSKI A.K. "On Maximally Graded Algebras and Walsh Functions", Reports on Math.Phys. (in press)

[3] KWÁSNIE'WSKI A.K. J.Phys.A:Math.Gen.19 (1986) 1469-1476

[4] KWAŚNILWSKI A.K. "Algebraic Properties of the 3-state Vector Potts Model" Reports on Math.Phys.(in press)

A.K. KWÁ́SNIE'WSKI

ISTITUIO DI FISICA; UNIVERSITA DI ROMA;

PIAZZALE ALDO HORO 2; I-00185 ROMA; ITALY 